\title{
Theory of perturbed equilibria for solving the Grad-Shafranov equation
}

\author{
L. E. Zakharov, A. Pletzer \\ June 21, 1999
}

\begin{abstract}
The theory of perturbed magnetohydrodynamic equilibria is presented for different formulations of the tokamak equilibrium problem. For numerical codes, it gives an explicit Newton scheme for solving the Grad-Shafranov equation subject to different constraints. The problem of stability of axisymmetric modes is shown to be a particular case of the equilibrium perturbation theory.
\end{abstract}

\section{Introduction}

Plasma equilibria lay at the fundamental level of magnetic confinement studies. All plasma processes including linear and early nonlinear stage of magnetohydrodynamic (MHD) instabilities, transport and plasma flows, waves, micro-instabilities and turbulence represent different kinds of deviations from MHD equilibrium, and, thus, require accurate calculations of equilibrium configurations $[1,2,3,4,5,6]$.

This paper describes a rigorous method for solving the nonlinear Grad-Shafranov equation given different sets of input profiles, e.g., $d p / d \Psi$ and $F d F / d \Psi, p$ and $q, p$ and $j_{\|}$, etc, where $p$ is the plasma pressure, $F$ the poloidal current, $q$ the safety factor, $j_{\|}$the averaged parallel component of the current density, and $\Psi$ the poloidal flux.

Because of the nonlinearity of the Grad-Shafranov (GSh) equation, all numerical methods for equilibrium calculations are iterative by nature [7] and can be subdivided into two classes: 1) Eulerian, which use a prescribed (e.g., rectangular or conformal to the plasma boundary) mesh calculation $[8,9,10,11,12,13,14]$ and 2) Lagrangian, which use curvilinear flux coordinates for equilibrium calculations [15, 16, 17, 18, 19, 20].

Eulerian methods have the advantage of being able to easily reproduce the 2-D geometry of complicated configurations [10]. They are widely used for simulations of equilibrium control in tokamaks [21] and for the interpretation of experimental magnetic measurements [22, 23, 24]. The disadvantage of those methods is in their limiting range of formulations of equilibrium problems, restricted essentially to a prescribed right hand side of the Grad-Shafranov (GSh) equation. Formulation requiring different input profiles can only be implemented with difficulty, due to the resulting frequent need of averaging over magnetic surfaces [25].

The use of flux coordinates allows to substantially extend the range of soluble problems. Essentially, any set of two 1-D functions of the radial coordinate, which uniquely determines the current density and the pressure profile $[2,3,26,27,16]$, can be used in flux coordinate based Lagrangian codes. The disadvantage of flux coordinate codes lies in their difficulty to treat free boundary plasmas with a separatrix.

Methods, which use flux coordinates, in their turn, can be subdivided into two categories: a) variational $[28,29,30,16,19]$ and inverse coordinate methods [31, 32, 17] and b) adaptive grid methods [15, 33, 18]. Variational codes solve equations where the unknowns are the flux coordinates themselves. Adaptive grid codes solve equilibrium equations in given curvilinear coordinates and then use the solution for advancing the coordinate system.

Practically all existing codes use a simple iteration method for solving the nonlinear GSh equation. Although this approach is sufficient in most of situations, there is still a demand for fast methods which can 
be used for extensive calculations of magnetic configurations in transport codes, for scrutinized studies of plasma stability (especially non-ideal), for equilibrium reconstruction from experimental measurements and for simulations of equilibrium control in real machines.

This paper describes a perturbative approach for solving the GSh equation using constraints that are specific to each formulation. The discrepancy between the nonlinear equation and its linear version then becomes a driving contribution. Such an approach is particularly adequate for the adaptive grid codes [15]. It is, however, more complicated to implement in variational or inverse coordinate codes due to the difficulty to linearize the equations in these codes (although some attempts have been made, [16]).

Together with an explicit algorithm which advances the coordinates, the theory of perturbed equilibria can be cast in a Newton scheme for solving non-linear tokamak equilibrium problems.

The use of perturbed equilibrium theory, however, finds applications beyond equilibrium calculations. In Sec. 5 we describe how the combination of flux conservation and adiabatic pressure constraints yields a formulation for axisymmetric stability problems [34, 35], thus, allowing equilibrium and axisymmetric stability calculations to be covered by the same codes.

The paper is organized in the following manner. Sect. 2 introduces the set of physical variables describing the equilibrium problems. Sect. 3 lists the relationships between the basic plasma profiles in equilibrium configurations. Sect. 4 describes the equations of perturbed equilibrium. Sect. 5 describes the coordinate advancing method, which is consistent with the choice of poloidal angle. Sect. 6 shows the connection between the Euler equation of ideal MHD stability and the theory of perturbed equilibrium. In the Summary, some results from the equilibrium code, based on the perturbation theory, are presented.

In this text, we use $m$, sec, $T, M A, M P a$ as units for length, time, magnetic field, current and pressure, respectively, as well as $V-s e c, M A / m^{2}$, etc. We will also use rationalized variables, typically present inside numerical codes and will distinguish them from the real physics variables by the bar sign.

\section{2-D magnetic fields and plasma currents in curvilinear coordi- nates}

Let $a, \tau, \phi$ be curvilinear coordinates in toroidal topology, where $a$ represents a radial coordinate, $\tau$ a poloidal angle and $\phi$ a toroidal angle (identical to the azimuthal angle in cylindrical coordinates). The cylindrical coordinates, $r, \phi, z$, can be expressed as

$$
r=r(a, \tau, \phi), \quad z=z(a, \tau, \phi) .
$$

We do not assume that the coordinates $a, \tau, \phi$ are flux coordinates. It is the goal of the adaptive method to adjust $(a, \tau, \phi)$ to magnetic flux tubes.

As an example, the transformation (2.1) can be expressed in the form of Fourier expansion in poloidal angle

$$
\begin{aligned}
& r=r_{0}^{c}(a)+2 \sum_{m=1}^{m \leq M}\left[r_{m}^{c}(a) \cos m \tau+r_{m}^{s}(a) \sin m \tau\right], \\
& z=z_{0}^{c}(a)+2 \sum_{m=1}^{m \leq M}\left[z_{m}^{c}(a) \cos m \tau+z_{m}^{s}(a) \sin m \tau\right] .
\end{aligned}
$$

This form is not unique and allows for different choices of poloidal angles. This issue has been intensively discussed in the literature, especially for stellarator equilibria (see, e.g., [36] and the references there). For tokamaks, there is a simple form of Fourier representation [4]

$$
\begin{aligned}
& r=r_{0}^{c}+2 \sum_{m=1}^{m \leq M}\left(r^{c} \cos m \tau+r^{s} \sin m \tau\right), \\
& z=z_{0}(a)+b(a) \sin \tau
\end{aligned}
$$


with only two harmonics in the $z$-coordinate. This form implies that any horizontal line $z=$ const intersects cross-section of the magnetic surface not more than two times. Despite of this limitation, this representation is suitable for practically all tokamak configurations.

In the radial direction, cubic splines or, e.g., Hermite polynomials can be used to represent the Fourier coefficients in the differentiable form, which is necessary for the calculation of the metric tensor $g_{i k}$

$$
d l^{2} \equiv g_{a a} d a^{2}+2 g_{a \tau} d a d \tau+g_{\tau \tau} d \tau^{2}+r^{2} d \phi^{2},
$$

specifying the element of length, which can easily be obtained from (2.1)

$$
g_{a a}=r_{a}^{\prime 2}+z_{a}^{\prime 2}, \quad g_{a \tau}=r_{a}^{\prime} r_{\tau}^{\prime}+z_{a}^{\prime} z_{\tau}^{\prime}, \quad g_{\tau \tau}=r_{\tau}^{\prime 2}+z_{\tau}^{\prime 2},
$$

where, e.g., $r_{a}^{\prime} \equiv \partial r / \partial a$. The poloidal Jacobian $D$ and the total Jacobian $\sqrt{g}$ are

$$
D \equiv \frac{D(r, z)}{D(a, \tau)}=r_{a}^{\prime} z_{\tau}^{\prime}-r_{\tau}^{\prime} z_{a}^{\prime}, \quad \sqrt{g}=r D,
$$

respectively.

The magnetic vector potential $\mathbf{A}$ is represented through its covariant components as

$$
\mathbf{A} \equiv A_{a} \nabla a+A_{\tau} \nabla \tau+A_{\phi} \nabla \phi=A_{a} \nabla a+\frac{\Phi}{2 \pi} \nabla \tau+\frac{\Psi}{2 \pi} \nabla \phi \equiv A_{a} \nabla a+\bar{\Phi} \nabla \tau+\bar{\Psi} \nabla \phi,
$$

where $\Phi$ is the toroidal flux trough the cross-section of coordinates $a=$ const, $\phi=$ const, while $\Psi$ is the poloidal flux between the cylindrical axis $r=0$ and the coordinate surface $a=$ const, $\tau=$ const. Note, that while the poloidal component $A_{\tau}=\bar{\Phi}$ in axisymmetric system can be chosen as a function of $a$ only, $\bar{\Phi}=\bar{\Phi}(a), \bar{\Psi}$ may be non-constant, $\bar{\Psi}=\bar{\Psi}(a, \tau)$, on the toroidal coordinate surface $a=$ const. We also introduce a periodic function $\eta$, related to the radial component of the vector potential

$$
A_{a} \equiv-\frac{d \bar{\Phi}}{d a} \eta
$$

In the case of flux coordinates, when $\Psi=\Psi(a)$, the function $\eta$ determines the transition from $\tau$ to the "straight field line" poloidal angle $\theta$

$$
\theta \equiv \tau+\eta
$$

Eqs. $(2.7,2.8)$ give a contravariant form for the magnetic field

$$
\begin{aligned}
& \mathbf{B}=\nabla \times \mathbf{A}=\bar{\Psi}_{\tau}^{\prime}(\nabla \tau \times \nabla \phi)+\bar{\Phi}^{\prime}\left(1+\eta_{\tau}^{\prime}\right)(\nabla a \times \nabla \tau)+\bar{\Psi}_{a}^{\prime}(\nabla a \times \nabla \phi), \\
& \mathbf{B} \equiv B^{a} \mathbf{e}_{a}+B^{\tau} \mathbf{e}_{\tau}+B^{\phi} \mathbf{e}_{\phi}, \quad \mathbf{e}_{a} \equiv r_{a}^{\prime} \mathbf{e}_{r}+z_{a}^{\prime} \mathbf{e}_{z}, \quad \mathbf{e}_{\tau} \equiv r_{\tau}^{\prime} \mathbf{e}_{r}+z_{\tau}^{\prime} \mathbf{e}_{z}, \quad B^{a}=\mathbf{B} \cdot \nabla a, \quad \ldots, \\
& r D B^{a}=\bar{\Psi}_{\tau}^{\prime}, \quad r D B^{\tau}=-\bar{\Psi}_{a}^{\prime}, \quad r D B^{\phi}=\bar{\Phi}^{\prime}\left(1+\eta_{\tau}^{\prime}\right) .
\end{aligned}
$$

The toroidal field, $B_{t o r}$, is related to the total poloidal current $F=F(a, \tau)$ in a simple way

$$
B_{t o r}=r B^{\phi}=\frac{\bar{F}}{r}, \quad \bar{F} \equiv 0.2 F=r B_{t o r},
$$

which gives the expression of the toroidal flux in terms of poloidal current

$$
\bar{\Phi}^{\prime}\left(1+\eta_{\tau}^{\prime}\right)=\frac{\bar{F} D}{r}, \quad \bar{\Phi}^{\prime}=\left(\frac{\bar{F} D}{r}\right)_{0} .
$$

Here and throughout the paper we use $(\ldots)_{0}$ to denote the zero harmonic in poloidal angle $\tau$, reserving the notation $\langle\ldots\rangle$ for conventional averaging between magnetic surfaces

$$
<f>\equiv \frac{\oint \sqrt{g} f d \tau}{\oint \sqrt{g} d \tau}=\frac{\oint \frac{f d \tau}{B_{p o l}}}{\oint \frac{d \tau}{B_{p o l}}}=\frac{(\sqrt{g} f)_{0}}{(\sqrt{g})_{0}} .
$$


The plasma current density $\mathbf{j}$ can be expressed in contravariant form,

$$
\begin{aligned}
& \mu_{0} \mathbf{j} \equiv \bar{j}^{a} \mathbf{e}_{a}+\bar{j}^{\tau} \mathbf{e}_{\tau}+\bar{j}^{\phi} \mathbf{e}_{\phi}, \quad \mu_{0} \equiv 0.4 \pi, \\
& r D \bar{j}^{a}=\bar{F}_{\tau}^{\prime}, \quad r D \bar{j}^{\tau}=-\bar{F}_{a}^{\prime}, \quad r D \bar{j}^{\phi}=\bar{J}_{a}^{\prime}+\nu_{\tau}^{\prime}
\end{aligned}
$$

in terms of the poloidal $\bar{F}(a, \tau)$ and toroidal $\bar{J}=0.2 J$ currents, and a periodic function $\nu_{\tau}^{\prime}$. The toroidal current $J$ is a function of $a$ only $J=J(a)$, while the function $\nu_{\tau}^{\prime}$ represents the oscillating part of the toroidal current density (related to the Pfirsch-Shlüter current).

Equilibrium equation

$$
\nabla p=\mathbf{j} \times \mathbf{B}
$$

gives the Grad-Shafranov expression for the toroidal component of the current density in terms of poloidal flux derivatives of the plasma pressure $p^{\prime}(\Psi)$ and $F F^{\prime}(\Psi)$

$$
\begin{aligned}
& \bar{j}_{\text {tor }}=r \frac{d \bar{p}}{d \bar{\Psi}}+\frac{1}{r} \bar{F} \frac{d \bar{F}}{d \bar{\Psi}}, \quad \bar{p} \equiv \mu_{0} p, \\
& \bar{J}^{\prime}+\nu_{\tau}^{\prime}=r D \frac{d \bar{p}}{d \bar{\Psi}}+\frac{D}{r} \bar{F} \frac{d \bar{F}}{d \bar{\Psi}}
\end{aligned}
$$

which, after substitution into toroidal component of Ampère's law

$$
\nabla \times \mathbf{B}=\mu_{0} \mathbf{j}
$$

leads to the Grad-Shafranov equation

$$
\Delta^{*} \bar{\Psi} \equiv \frac{\partial^{2} \bar{\Psi}}{\partial r^{2}}-\frac{1}{r} \frac{\partial \bar{\Psi}}{\partial r}+\frac{\partial^{2} \bar{\Psi}}{\partial z^{2}}=-r^{2} \frac{d \bar{p}}{d \bar{\Psi}}-\bar{F} \frac{d \bar{F}}{d \bar{\Psi}}
$$

We introduce the notation

$$
P(\Psi) \equiv \frac{d \bar{p}}{d \bar{\Psi}}, \quad T(\Psi) \equiv \bar{F} \frac{d \bar{F}}{d \bar{\Psi}}
$$

for the two 1-D functions in the RHS of this equation. In the curvilinear coordinates, the GSh equation can be written in the form

$$
\mathcal{L} \bar{\Psi}=-r D P-\frac{D}{r} T
$$

where $\mathcal{L}$ is a partial differential operator

$$
\mathcal{L} \equiv \frac{\partial}{\partial a} \frac{g_{\tau \tau}}{r D} \frac{\partial}{\partial a}-\frac{\partial}{\partial a} \frac{g_{a \tau}}{r D} \frac{\partial}{\partial \tau}-\frac{\partial}{\partial \tau} \frac{g_{a \tau}}{r D} \frac{\partial}{\partial a}+\frac{\partial}{\partial \tau} \frac{g_{a a}}{r D} \frac{\partial}{\partial \tau}=\frac{D}{r} \Delta^{*} .
$$

We designate the geometric coefficients present in the differential GSh operator by

$$
K \equiv \frac{g_{\tau \tau}}{r D}, \quad G_{a \tau} \equiv \frac{g_{a \tau}}{r D}, \quad G_{a a} \equiv \frac{g_{a a}}{r D},
$$

and in the RHS by

$$
L \equiv \frac{D}{r}, \quad V \equiv D r
$$




\section{Basic plasma profiles in equilibrium configurations}

In this section, assuming that $a=a^{*}, \tau, \phi$ are already equilibrium flux coordinates

$$
\bar{\Psi}=\bar{\Psi}\left(a^{*}\right), \quad \bar{p}=\bar{p}\left(a^{*}\right), \quad \bar{F}=\bar{F}\left(a^{*}\right),
$$

we give expressions for typical 1-D plasma profiles and their relationships with the RHS of the GSh equation. They can be used as input equilibrium profiles in the context of transport and stability studies.

Denoting the magnetic axis radius by $R_{0}$, we can express the toroidal current density

$$
\bar{j}_{\text {tor }} \equiv \bar{j}_{s} \frac{R_{0}}{r}+\bar{j}_{p}\left(\frac{r}{R_{0}}-\frac{R_{0}}{r}\right),
$$

in terms of two 1-D functions $\bar{j}_{s}\left(a^{*}\right)$ and $\bar{j}_{p}\left(a^{*}\right)$, which have the dimensions of a current density:

$$
\bar{j}_{p} \equiv P R_{0}, \quad \bar{j}_{s} \equiv \frac{T}{R_{0}}+P R_{0} .
$$

At the magnetic axis we have $\bar{j}_{\text {tor }}=\bar{j}_{s}$.

The total current $\bar{J}$ through the cross-section of the magnetic surface is

$$
\bar{J}=\int_{0}^{a^{*}}\left(T L_{0}+P V_{0}\right) d a, \quad L_{0} \equiv(L)_{0}, \quad V_{0} \equiv(V)_{0} .
$$

The GSh equation, written in flux coordinates, then reduces to

$$
\frac{\partial\left(K \bar{\Psi}^{\prime}\right)}{\partial a^{*}}-\frac{\partial\left(G_{a^{*} \tau} \bar{\Psi}^{\prime}\right)}{\partial \tau}=-T L-P V
$$

which, after averaging

$$
\left(K_{0} \bar{\Psi}^{\prime}\right)^{\prime}=-T L_{0}-P V_{0}, \quad K_{0} \equiv(K)_{0}
$$

gives an expression of the toroidal current $\bar{J}$

$$
\bar{J}=-K_{0} \bar{\Psi}^{\prime}
$$

in terms of $\bar{\Psi}^{\prime}$. The toroidal flux $\bar{\Phi}$ through the cross-section of the magnetic surface is

$$
\bar{\Phi}=\int_{0}^{a^{*}} \bar{F} L_{0} d a,
$$

whereas the safety factor $q$ has the form

$$
q=-\frac{\bar{\Phi}^{\prime}}{\bar{\Psi}^{\prime}}=-\frac{\bar{F} L_{0}}{\bar{\Psi}^{\prime}}
$$

The slow time evolution is described by the parallel component of Ohm's law (taken here in its simplest form $[1,2])$

$$
\mathbf{B} \cdot \mathbf{j}=\bar{\sigma} \mathbf{B} \cdot \mathbf{E}, \quad \mathbf{E}=-\nabla \phi_{E}-\frac{\partial \mathbf{A}}{\partial t}, \quad \bar{\sigma} \equiv 10^{-6} \sigma,
$$

where $\mathbf{E}$ is the electric field, $\phi_{E}$ is the scalar potential of electric field, $\sigma$ is electrical conductivity (the factor $10^{-6}$ appeared in the rationalized $\bar{\sigma}$ because of our units of $M A / \mathrm{m}^{2}$ for the current density). After averaging over the layer between magnetic surfaces (2.13) we get

$$
<\mathbf{B} \cdot \mathbf{j}\rangle=-\bar{\sigma}\left\langle\mathbf{B} \cdot \frac{\partial \mathbf{A}}{\partial t}\right\rangle,
$$


Eq. (3.10) gives the equilibrium evolution equation in the form of

$$
\frac{\partial \bar{\Psi}^{\prime}}{\partial t}+q \frac{\partial \bar{\Phi}}{\partial t}=-\frac{<\mathbf{B} \cdot \mathbf{j}>}{\bar{\sigma}<\mathbf{B} \cdot \nabla \phi>} .
$$

We introduce a profile $j_{\|}$associated with Ohm's law

$$
j_{\|} \equiv \frac{<\mathbf{B} \cdot \mathbf{j}>}{R_{0}<\mathbf{B} \cdot \nabla \phi>}
$$

which has the dimension of a current density and, at the magnetic axis, is equal to the value of the physical current density. In flux coordinates

$$
j_{\|}=\frac{1}{R_{0} L_{0}}\left(\bar{J}^{\prime}+\frac{K_{0} \bar{\Psi}^{\prime} \bar{F}^{\prime}}{\bar{F}}\right)=\frac{1}{R_{0} L_{0}}\left(L_{0} T+V_{0} P+\frac{K_{0} \bar{\Psi}^{\prime} \bar{F}^{\prime}}{\bar{F}}\right),
$$

In terms of this profile, the time evolution equation then becomes

$$
\frac{\partial \bar{\Psi}^{\prime}}{\partial t}+q \frac{\partial \bar{\Phi}}{\partial t}=-\frac{R_{0} j_{\|}}{\bar{\sigma}} .
$$

It can also be written in the form of a diffusion equation for $\bar{\Psi}$

$$
\frac{\partial \bar{\Psi}^{\prime}}{\partial t}+q \frac{\partial \bar{\Phi}}{\partial t}=\frac{1}{L_{0} \bar{\sigma}}\left[\left(K_{0} \bar{\Psi}^{\prime}\right)^{\prime}-\frac{K_{0} \bar{\Psi}^{\prime} \bar{F}^{\prime}}{\bar{F}}\right] .
$$

The 1-D profiles mentioned here essentially exhaust the list of typical input profiles for equilibrium calculations. Nevertheless, there are other possible non-conventional profiles, e.g., the pitch angle $\iota$ of poloidal magnetic field, related to the MSE measurements in tokamaks [37, 38],

$$
\iota \equiv \frac{B_{p o l}}{B_{t o r}}
$$

as a function of the major radius $r$, or bootstrap fraction of the current density.

For different input profiles, it is necessary to use different forms of averaged GSh equation (3.6) in order to generate the zeroth order approximation for the solution. We list here some of them for a typical set of input profiles:

1. For given $P$ and $T$, the first order differential equations:

$$
J^{\prime}=L_{0} T+V_{0} P, \quad \bar{\Psi}^{\prime}=-\frac{J}{K_{0}}, \quad \bar{F} \bar{F}^{\prime}=T \bar{\Psi}^{\prime}, \text { and } \quad \bar{p}^{\prime}=P \bar{\Psi}^{\prime}
$$

determine all necessary equilibrium profiles.

2. For given $\bar{j}_{\|}$and $P$, we use

$$
\left(\frac{\bar{J}}{\bar{F}}\right)^{\prime}=\frac{L_{0} R_{0} \bar{j}_{\|}}{\bar{F}}, \quad \bar{\Psi}^{\prime}=-\frac{J}{K_{0}}, \quad\left(1+\frac{\bar{J}^{2}}{K_{0} L_{0} \bar{F}^{2}}\right) \bar{F} \bar{F}^{\prime}=\frac{V_{0} \bar{J}}{K_{0} L_{0}} P-\frac{\bar{J}}{K_{0}} R_{0} \bar{j}_{\|}, \quad \bar{p}^{\prime}=P \bar{\Psi}^{\prime} .
$$

3. For given $\bar{j}_{\|}$and $\bar{p}$ (an appropriate choice for transport codes), the equations are similar to the previous set

$$
\left(\frac{\bar{J}}{\bar{F}}\right)^{\prime}=\frac{L_{0} R_{0} \bar{j}_{\|}}{\bar{F}} \quad \bar{\Psi}^{\prime}=-\frac{J}{K_{0}}, \quad\left(1+\frac{\bar{J}^{2}}{K_{0} L_{0} \bar{F}^{2} 2}\right) \bar{F} \bar{F}^{\prime}=-\frac{V_{0}}{L_{0}} \bar{p}^{\prime}-\frac{\bar{J}}{K_{0}} R_{0} \bar{j}_{\|} .
$$


4. If the rotational transform $\mu=1 / q$ is specified, then either

$$
\left(1+K_{0} L_{0} \mu^{2}\right) \bar{F}^{\prime}+\mu\left(K_{0} L_{0} \mu\right)^{\prime} \bar{F}=\mu \frac{V_{0}}{L_{0}} P
$$

should be used if $P$ is given, or

$$
\left(1+K_{0} L_{0} \mu^{2}\right) \bar{F} \bar{F}^{\prime}+\mu\left(K_{0} L_{0} \mu\right)^{\prime} \bar{F}^{2}=-\frac{V_{0}}{L_{0}} \bar{p}^{\prime},
$$

if $p$ is given. Other profiles can be found from the algebraic relationships of this section.

5. Given $\bar{\Psi}$ and either $\bar{p}$ or $P$ (another appropriate choice for interfacing with transport codes) leads simply to

$$
L_{0} \bar{F} \bar{F}^{\prime}=-\left(K_{0} \bar{\Psi}^{\prime}\right)^{\prime}-V_{0} P=-\left(K_{0} \bar{\Psi}^{\prime}\right)^{\prime}-V_{0} \frac{\bar{p}^{\prime}}{\bar{\Psi}^{\prime}} .
$$

\section{Perturbed Grad-Shafranov equation}

The Grad-Shafranov equation (2.20) implies that there is a degree of freedom in the choice of the two 1-D profiles (e.g., $P, T$ or $p, j_{\|}$) uniquely determining the pressure and current distributions. In fact, there is also a degree of freedom in the choice of the radial variable $a$ which labels the magnetic surfaces (e.g., $a=\sqrt{\Phi / \bar{\Phi}_{b}}$, where $\bar{\Phi}_{b}$ is the total toroidal flux through the plasma cross-section). Thus, besides the boundary conditions, there are in total three 1-D constraints for solving the GSh equation.

Assuming that the coordinate system $a, \tau$ is close to the real (target) flux coordinates, $a^{*}, \tau$,

$$
a=a^{*}+\xi(a, \tau),
$$

we can write the transition from the current $r=r(a, \tau), z=z(a, \tau)$ coordinates to the target flux coordinates $r=r^{*}\left(a^{*}, \tau\right), z=z^{*}\left(a^{*}, \tau\right)$ in explicit form as

$$
r^{*} \equiv r\left(a^{*}+\xi, \tau\right) \simeq r\left(a^{*}, \tau\right)+r_{a}^{\prime} \xi, \quad z^{*} \equiv z\left(a^{*}+\xi, \tau\right) \simeq z\left(a^{*}, \tau\right)+z_{a}^{\prime} \xi
$$

in linear approximation. The displacement $\xi$ of the coordinates can be found from the flux coordinate equation

$$
\bar{\Psi}(a, \tau) \equiv \bar{\Psi}^{0}(a)+\psi(a, \tau)=\text { const },
$$

where $\bar{\Psi}^{0}(a)$ is the main order approximation for the solution, which can be obtained by solving the averaged equations of the previous section, and $\psi$ the (unknown) linear perturbation of the poloidal flux function. In linear approximation, Eq. (4.3) gives a relationship between the displacement $\xi$ and the perturbation of the poloidal flux

$$
\bar{\Psi}^{\prime 0} \xi+\psi=\text { const. }
$$

In the displacement $\xi$ it is necessary to distinguish between its average, $\xi_{0}$, and oscillatory, $\xi_{\sim}$, parts

$$
\xi=\xi_{0}(a)+\xi_{\sim}(a, \tau) .
$$

Eq. (4.4), in fact, determines only the oscillatory part of the displacement

$$
\xi_{\sim}=-\frac{\psi_{\sim}}{\bar{\Psi}^{\prime 0}},
$$

since the constant in the RHS of Eq. (4.4) is unknown. Equation (4.6) is equivalent to the condition of absence of radial component of the magnetic field $B \cdot \nabla a^{*}=0$, written in linearized form. 
The average part of displacement $\xi_{0}$ is determined by the labeling of the magnetic surfaces. For example, if the poloidal flux $\bar{\Psi}$ is chosen as the radial coordinate $a$, then

$$
\xi_{0}=\frac{a-\bar{\Psi}^{0}-\psi_{0}}{\bar{\Psi}^{\prime 0}} .
$$

Note, that because $\bar{\Psi}^{0}$ is calculated by solving the 1-D equations of the previous section, it is easy to adjust coordinates in a way that $a=\bar{\Psi}^{0}$. In this case

$$
\xi_{0}=-\frac{\psi_{0}}{\bar{\Psi}^{\prime 0}}, \quad \xi=-\frac{\psi}{\bar{\Psi}^{\prime 0}}
$$

and the displacement $\xi$ is "frozen" into poloidal flux as in ideal stability theory.

If the normalized toroidal flux serves as the radial coordinate, $a \equiv \bar{\Phi} / \bar{\Phi}_{b}$, then

$$
\xi_{0}=\frac{a \bar{\Phi}_{b}-\bar{\Phi}}{\bar{\Phi}^{\prime}} .
$$

If square root $\sqrt{\bar{\Phi} / \bar{\Phi}_{b}}$ of the normalized toroidal flux is chosen as $a$, then

$$
\xi_{0}=2 \frac{a \sqrt{\bar{\Phi} \bar{\Phi}_{b}}-\bar{\Phi}}{\bar{\Phi}^{\prime}} .
$$

Other choices can be covered in analogous way.

Now, it is possible to linearize the Grad-Shafranov equation

$$
\Delta^{*} \bar{\Psi}=-r^{2} P\left(a^{*}\right)-T\left(a^{*}\right)
$$

Its differential operator is already linear and only the RHS has to be linearized

$$
P\left(a^{*}\right)=P(a)-P^{\prime} \xi+\delta P, \quad T\left(a^{*}\right)=T(a)-T^{\prime} \xi+\delta T,
$$

where in both equations the first term in the RHS represents the main approximation, while the second term is the convective perturbation. The third term represents the non-convective part of perturbations of $\delta P$ or $\delta T$ and is determined by linearization of the constraints on the two inputs profiles, as shown below. Using Eqs. (4.12), the perturbed GSh equation can be written as

$$
\Delta^{*} \bar{\Psi}=-r^{2} P(a)-T(a)+r^{2} P^{\prime} \xi+T^{\prime} \xi-r^{2} \delta P-\delta T,
$$

or in the curvilinear coordinates as

$$
\mathcal{L} \bar{\Psi}=-V P-L T+V P^{\prime} \xi+L T^{\prime} \xi-V \delta P-L \delta T
$$

The conventional simple iteration scheme [7] for solving GSh equation used in most equilibrium codes, takes into account only the first two terms in the RHS of Eq. (4.13). The addition of linear terms into the RHS accelerates convergence of solving the nonlinear GSh equaton.

Now, let us consider the perturbations $\delta P$ and $\delta T$ in Eq.(4.13), which depend on the input profiles. If the input profiles are $P(a)=d \bar{p} / d \bar{\Psi}$ and $T(a)=\bar{F} d \bar{F} / d \bar{\Psi}$, then $\delta P=0$ and $\delta T=0$.

Typically, e.g., in interfacing with the transport codes, pressure profile $\bar{p}(a)$, rather than $P$ is given as a function of $a$. In this case,

$$
\delta P \equiv \delta \frac{\bar{p}^{\prime}}{\bar{\Psi}^{\prime 0}}=-\frac{\bar{p}^{\prime}}{\bar{\Psi}^{\prime 0}} \frac{\delta \psi_{0}^{\prime}}{\bar{\Psi}^{\prime 0}}=-P \frac{\delta \psi_{0}^{\prime}}{\bar{\Psi}^{\prime 0}},
$$

where $\delta \psi_{0}$ is the non-convective part of the perturbation of $\psi_{0}$ :

$$
\delta \psi_{0} \equiv \psi_{0}+\bar{\Psi}^{\prime 0} \xi_{0}
$$


In more complicated cases, the relationship between $\delta P$ and $\delta T$ and the input profiles contain certain averaged metric coefficients, such as $L_{0}, V_{0}, K_{0}$. Their perturbations can be obtained by using the coordinate transformation (4.2). It is straightforward to see that

$$
\delta D=(D \xi)_{a}^{\prime}
$$

Consequently,

$$
\delta V=\delta(r D)=(V \xi)_{a}^{\prime}, \quad \delta L=\delta\left(\frac{D}{r}\right)=(L \xi)_{a}^{\prime} .
$$

The perturbation of the metric coefficient $K$

$$
\delta K=\frac{g_{\tau \tau}}{r D}=(K \xi)_{a}^{\prime}-2 K \xi_{a}^{\prime}+2 G_{a \tau} \xi_{\tau}^{\prime}
$$

can be expressed in terms of the displacement $\xi$.

Now, let us consider typical input profiles, related to the current density, taken as exemples in the previous section. If $q(a)$ ( or $\mu=1 / q$ ) is given as an input profile, the perturbation of $\bar{F}$ can be obtained from formula $(3.9)$

$$
\delta \bar{F} L_{0}=-\bar{F} \delta L_{0}-q \delta \psi_{0}^{\prime}=-\bar{F}(L \xi)_{a 0}^{\prime}-q \delta \psi_{0}^{\prime} .
$$

This yields $\delta T$ in the form of the second order integro-differential operator

$$
\delta T=\frac{(\bar{F} \delta \bar{F})^{\prime}}{\bar{\Psi}^{\prime 0}}-T \frac{\delta \psi_{0}^{\prime}}{\bar{\Psi}^{\prime 0}}=\frac{1}{\bar{\Psi}^{\prime 0}}\left(-\frac{\bar{F}^{2}(L \xi)_{a 0}^{\prime}}{L_{0}}+\bar{F}^{2} \frac{\delta \psi_{0}^{\prime}}{\bar{\Psi}^{\prime 0}}\right)_{a}^{\prime}-T \frac{\delta \psi_{0}^{\prime}}{\bar{\Psi}^{\prime 0}}
$$

If the radial coordinate is chosen as a function of either the poloidal $\bar{\Psi}$ or toroidal $\bar{\Phi}$ flux (typically the case in the transport codes), then $\delta \psi_{0}=0$ and there is only one term left in the RHS of this equation.

When expression (4.21) for $\delta T$ is substituted into perturbed GSh equation (4.13), it can be noticed that because of the strong toroidal magnetic field of the tokamak, the differential operator coming from $\delta T$ and containing a big factor $\bar{F}^{2}$ will be dominant. By taking this into account, it is possible to use a simplified approximate condition

$$
(L \xi)_{0}=0
$$

instead of the rigorous expression (4.21) for $\delta T$. In order to do this in an effective way, let us distinguish between the averaged and oscillatory parts of the GSh equation. Then, $\delta T$ can be kept only in the averaged component of equation (4.13)

$$
(\mathcal{L} \bar{\Psi})_{0}=-V_{0} P-L_{0} T-V_{0} \delta P-L_{0} \delta T+P^{\prime}(V \xi)_{0}+T^{\prime}(L \xi)_{0},
$$

and excluded from the oscillating part of this equation

$$
\mathcal{L} \bar{\Psi}-\frac{L}{L_{0}}(\mathcal{L} \bar{\Psi})_{0}=\left(V_{0} \frac{L}{L_{0}}-V\right)(P+\delta P)+P^{\prime}\left[(V \xi)_{0} \frac{L}{L_{0}}-V \xi\right]+T^{\prime}\left[(L \xi)_{0} \frac{L}{L_{0}}-L \xi\right] .
$$

This separation gives more flexibility in the treatment of constraints. On the one hand, the 1-D equation (4.23) and 2-D equation (4.24) can be considered as a system of equations if both $\delta P$ and $\delta T$ are provided by other considerations. On the other hand, the entire averaged equation (4.23), which contains the term $\delta T$, can be replaced with simpler constraints, such as, e.g., the algebraic expression (4.22). The simplest case of similar constraints corresponds to formulation 5 in the previous section, where $\bar{\Psi}$ is specified as an input profile and there is no needs to derive $\delta T$.

The rigorous expressions for $\delta T$ can be obtained in a similar way when $\bar{j}_{\|}$is the input profile. Because $\delta T$ affects only the convergence rate, it is possible to use some reasonable simplifications in $\delta T$, and to drop, 
for example, terms containing the ratio between poloidal and toroidal magnetic fields in tokamaks. For given $\bar{j}_{\|}$(formulations 2, 3 in the previous section), we get a simple expression for $\delta T$

$$
\delta T=\delta R_{0} j_{\|}-\frac{V_{0}}{L_{0}} \delta P-\frac{(V \xi)_{a 0}^{\prime}}{L_{0}} P+\frac{V_{0}(L \xi)_{a 0}^{\prime}}{L_{0}^{2}} P
$$

The perturbed Grad-Shafranov equation (4.13) or (4.14) together with Eq.(4.6) determine the flux function $\bar{\Psi}$ and the displacement of coordinates $\xi$, given boundary conditions and three additional constraints specifying $\xi_{0}, \delta P$, and $\delta T$. The coordinate system can then be advanced explicitly. This process can be repeated until sufficient accuracy is reached. In this form, it constitutes the Newton method for solving nonlinear equilibrium equations.

\section{Advancing the coordinate system}

The perturbed equilibrium equation is written in terms of the unknown poloidal flux $\bar{\Psi}=\bar{\Psi}^{0}+\psi$ and the radial plasma displacement $\xi$. The relation between $\psi$ and $\xi$ is algebraic, given by (4.6) and either one of (4.7)-(4.10). Once the solution is obtained, it should be used for advancing the coordinate system toward the flux coordinates. A straigtforward algorithm (4.2) for advancing the coordinates does not preserve the specific choice of the poloidal angle, e.g. (2.3). A more flexible scheme containing both the radial $\xi$ and tangential $\sigma$ displacements

$$
r \rightarrow r+r_{a}^{\prime} \xi+r_{\tau}^{\prime} \sigma, \quad z \rightarrow z+z_{a}^{\prime} \xi+z_{\tau}^{\prime} \sigma
$$

must be applied. In linear approximation the tangential displacements is arbitrary and, thus, can be used to satisfy constraints on the poloidal angle in the coordinate representation.

For the poloidal angle determined by Eq. (2.3), the tangential displacement should satisfy equation

$$
\delta z=\delta z_{0}+\delta b \sin \tau=z_{a}^{\prime} \xi+b \sigma \cos \tau,
$$

in order to preserve the specific form of Fourier expansion of the $z$-coordinate. Here $\delta z_{0}, \delta b$ are correction to its Fourier coefficients, which can be determined from

$$
\delta z_{0}+\delta b=\left.\left(z_{0}^{\prime}+b^{\prime}\right) \xi\right|_{\tau=\pi / 2}, \quad \delta z_{0}-\delta b=\left.\left(z_{0}^{\prime}-b^{\prime}\right) \xi\right|_{\tau=-\pi / 2} .
$$

This leads to the following equation for the perturbation $\delta r$

$$
b \cos \tau \delta r=D \xi+r_{\tau}^{\prime}\left(\delta z_{0}+\delta b \sin \tau\right),
$$

which can be easily solved in Fourier space for the Fourier coefficients of $\delta r$.

Note, that for the coordinate system, which is defined by Eqs. (2.3), the vertical semiaxis $b$ can be chosen as a radial coordinate $a$ with a simple geometrical interpretation. In this case, $\xi_{0}$ is determined from the condition $\delta b=0$

$$
\xi_{0}=-\frac{z_{0}^{\prime}}{2 b^{\prime}}\left(\left.\xi_{\sim}\right|_{\tau=\pi / 2}-\left.\xi_{\sim}\right|_{\tau=-\pi / 2}\right)-\frac{1}{2}\left(\left.\xi_{\sim}\right|_{\tau=\pi / 2}+\left.\xi_{\sim}\right|_{\tau=-\pi / 2}\right) .
$$

Explicit coordinate advancing algorithm is consistent with the Newton approach for solving the entire equilibrium problem.

\section{Perturbed equilibria and ideal energy principle}

The perturbed equilibrium equations, written in the previous section, cover arbitrary (axisymmetric) perturbations. As a particular case, they should reproduce the marginally stable perturbations of the ideal 
stability theory if the condition of frozen magnetic field is imposed as the constraint. Indeed, it is easy to see how this happens.

The local adiabatic equation of state, used in the ideal stability theory, can be expressed in our notation as

$$
p(r D)^{\gamma_{0}}=p V_{0}^{\gamma_{0}}=\text { const }
$$

where $\gamma_{0}$ is the adiabatic constant. Based on this expression, the non-convective change in the pressure $\delta \bar{p}$ can be obtained as

$$
\delta \bar{p}=-\gamma_{0} \bar{p} Y_{0}, \quad Y_{0} \equiv \frac{(V \xi)_{0}^{\prime}}{V_{0}},
$$

which leads to the following expression for $\delta P$

$$
\delta P=\frac{\delta \bar{p}^{\prime}}{\bar{\Psi}^{\prime}}=-\frac{\left(\gamma_{0} \bar{p} Y_{0}\right)^{\prime}}{\bar{\Psi}^{\prime}} .
$$

The non-convective perturbation of $\bar{F}$ is determined by the frozen magnetic field condition. Assuming, that the radial coordinate is frozen into the toroidal flux $\delta \bar{\Phi}=0$

$$
\delta \bar{\Phi}^{\prime}=\delta\left(L_{0} \bar{F}\right)=0
$$

the perturbation $\delta \bar{F}$ can be found in the form

$$
L_{0} \delta \bar{F}=-\bar{F}(L \xi)_{0}^{\prime}, \quad \delta \bar{F}^{2}=2 \bar{F} \delta \bar{F}=-2 \bar{F}^{2} X_{0}, \quad X_{0} \equiv \frac{(L \xi)_{0}^{\prime}}{L_{0}},
$$

which leads to the following expression for $\delta T$

$$
\delta T \equiv \frac{\left(\delta \bar{F}^{2}\right)^{\prime}}{2 \bar{\Psi}^{\prime}}=-\frac{\left(\bar{F}^{2} X_{0}\right)^{\prime}}{\bar{\Psi}^{\prime}}
$$

With these $\delta P$ and $\delta T$ in the RHS, the perturbed equilibrium equation (4.13), when applied to an equilibrium configuration, becomes identical to the Euler equation, which can be obtained from the energy principle for (marginal, $\gamma=0$ ) stability of the ideal axisymmetric modes [34, 35], i.e.,

$$
\Delta^{*} \psi=r^{2} \frac{\left(\gamma_{0} \bar{p} Y_{0}\right)^{\prime}}{\bar{\Psi}^{\prime}}+\frac{\left(\bar{F}^{2} X_{0}\right)^{\prime}}{\bar{\Psi}^{\prime}}+r^{2} P^{\prime} \xi+T^{\prime} \xi
$$

For ideal plasmas, the displacement of coordinates $\xi$ coincides with the radial (contravariant) component of the plasma displacement in stability theory.

The frozen condition of the magnetic flux is a natural constraint for the fast evolution of equilibrium, e.g., when the equilibrium control is lost and the plasma motion is determined by the eddy currents in the resistive surroundings. Adiabaticity of the pressure is not well justified for those problems. In fact, in experiments, pressure losses may occur on a much faster time scale. For this reason, a more simple, prescribed pressure model as described in the previous section seems to be more adequate. While conventional ideal stability theory is not applicable to this case, the perturbed equilibrium theory can describe such a situation.

The fact that the ideal marginal stability condition can be reproduced by the perturbation theory, allows to combine in a natural way both equilibrium and axisymmetric stability problems in the same numerical code. In the conventional practice, these two problems are, typically, treated as separate.

\section{Summary}

The theory of perturbed equilibrium gives a rigorous algorithm for solving different equilibrium problems in tokamaks. 
Its use leads to fast numerical codes, which find application in transport simulations where the magnetic configuration evolves gradually. In these circumstances, the Newton scheme is most effective and requires, typically, only two iterations. In high beta cases, the Newton scheme eliminates oscillations, which the simple iterations scheme may exhibit.

One of such a code, ESC, has been developed in the Princeton Plasma Physics Laboratory in 1994 and has been intensively used for treatment of the Tokamak Fusion Test Reactor (TFTR) data. It is also applicable for low aspect ratio equilibria. Fig. 1 shows the effectiveness of the coordinate system (2.3). The analytical Soloviev solution for the National Spherical Torus Experiment (NSTX) aspect ratio is reproduced accurately with only 5 harmonics. Flexibility of the ESC code in accepting different input profiles facilitates its interface and comparison with other codes. Figs. 2,3 shows the comparison of ESC with VMEC [29] and JSOLVER [15] for NSTX equilibrium with $\beta=8 \%$ and $\beta=16 \%$. While VMEC shows small deviations form ESC, the accuracy of JSOLVER (with a fixed number of nodes $65 \times 65$ ) gradually deteriorates with increasing pressure. High- $\beta$ equilibrium with $\beta=45 \%$ on Fig. 4 was calculated by ESC, while two other codes failed at the level of $\beta=20 \%$. More details of ESC numerical technique will be published separately.

Although the present theory has been developed for axisymmetric configuration, its ideas are also applicable to 3-D MHD equilibrium codes, where the issue of numerical efficiency becomes critical.

\section{Acknowledgments}

This work was supported by the U.S. Department of Energy Contract No. DE-AC02-76-CHO-3073.

\section{References}

[1] V. D. Shafranov, Reviews of Plasma Physics (Consultant Bureau, New York, 1966), Vol. 2, p. 103.

[2] H. Grad and J. Hogan, Phys. Rev. Letters 24, 1337 (1970).

[3] H. Grad, P. N. Hu, and D. C. Stevens, Proc. Nat. Acad. Sci. 72, 3789 (1975).

[4] L. E. Zakharov and V. D. Shafranov, Reviews of Plasma Physics (Consultant Bureau, New York-London, 1981), Vol. 11, p. 153.

[5] R. R. Khairutdinov and V. E. Lukash, Journal of Computational Physics 109, 193 (1993).

[6] D. A. Ernst et al., Nucl. Fusion 38, 13 (1998).

[7] K. Lackner, Computer Physics Communications 12, 33 (1976).

[8] J. D. Callen and R. A. Dory, Phys. Fluids 15, 1523 (1972).

[9] W. Feneberg and K. Lackner, Nucl. Fusion 13, 549 (1973).

[10] F. J. Helton and T. S. Wang, Nucl. Fusion 18, 1523 (1978).

[11] J. L. Johnson et al., Journal of Computational Physics 32, 212 (1979).

[12] J. P. Goedbloed, Computer Physics Communications 24, 311 (1981).

[13] J. Blum, J. L. Foll, and B. Thooris, Computer Physics Communications 24, 235 (1981).

[14] H. Lütjens, A. Bondesons, and A. Roy, Computer Physics Communications 69, 287 (1992).

[15] J. DeLucia, S. C. Jardin, and A. M. M. Todd, Journal of Computational Physics 37, 183 (1980). 
[16] K. M. Ling and S. C. Jardin, Journal of Computational Physics 58, 300 (1985).

[17] R. Gruber, R. Iacono, and F. Troyon, Journal of Computational Physics 73, 168 (1987).

[18] G. T. A. Huysmans, J. P. Goedbloed, and W. Kerner, in Proc. CP90 Conf. on Comp. Phys. Proc. (World Scientific Publ. Co., 1991), p. 371.

[19] B. Turkington, A. Lifschitz, A. Eydeland, and J. Spruck, Journal of Computational Physics 106, 269 (1993).

[20] G. O. Ludwig, Plasma Phys. and Controlled Fusion 39, 2021 (1997).

[21] R. Albanese and F. Villone, Nucl. Fusion 38, 723 (1998).

[22] L. L. Lao, H. E. S. J. R. and. D. Stambaugh, A. G. Kellman, and W. Pfeiffer, Nucl. Fusion 25, 1611 (1985).

[23] J. Blum et al., Nucl. Fusion 30, 1475 (1990).

[24] J. R. Ferron et al., Nucl. Fusion 38, 1055 (1997).

[25] L. LoDestro and L. D. Pearlstein, Phys. of Plasmas 1, 90 (1994).

[26] J. F. Clark and D. J. Sigmar, Phys. Rev. Letters 38, 70 (1977).

[27] S. P. Hirshman and S. C. Jardin, Phys. Fluids 22, 731 (1979).

[28] V. D. Khait, Soviet Journal of Plasma Physics 6, 476 (1980).

[29] L. L. Lao, S. P. Hirshman, and R. M. Wieland, Phys. Fluids 24, 1431 (1981).

[30] L. L. Lao, Computer Physics Communications 31, 201 (1984).

[31] P. Vabishevich, L. M. Degtyarev, and A. P. Favorski, Soviet Journal of Plasma Physics 4, 554 (1978).

[32] L. M. Degtyarev and V. V. Drozdov, Computer Physics Reports 2, 341 (1985).

[33] L. M. Degtyarev et al., International Journal of Modern Physics C 2, 30 (1991).

[34] M.-S. Chu and R. L. Miller, Phys. Fluids 21, 817 (1978).

[35] S. W. Haney, L. D. Pearlstein, R. H. Bulmer, and J. P. Freidberg, Plasma Physics Reports 23, 789 (1997).

[36] S. P. Hirshman and J. Breslau, Phys. of Plasmas 5, 2664 (1998).

[37] S. P. Hirshman et al., Phys. of Plasmas 1, 2277 (1994).

[38] S. H. Batha et al., Nucl. Fusion 36, 1133 (1996). 


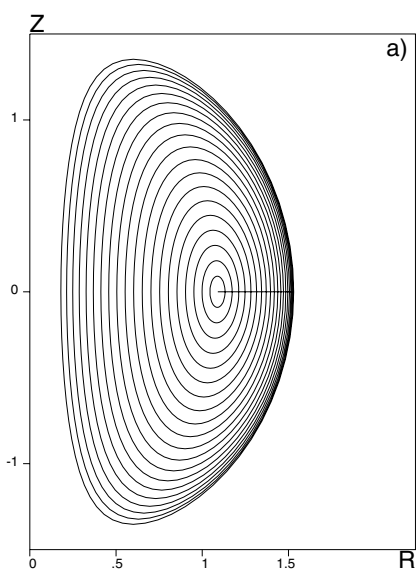

a)

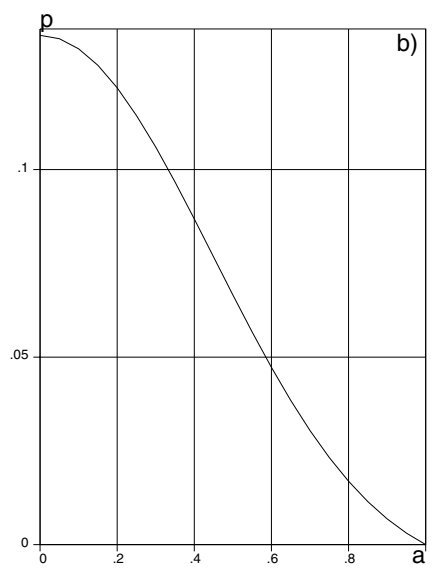

b)

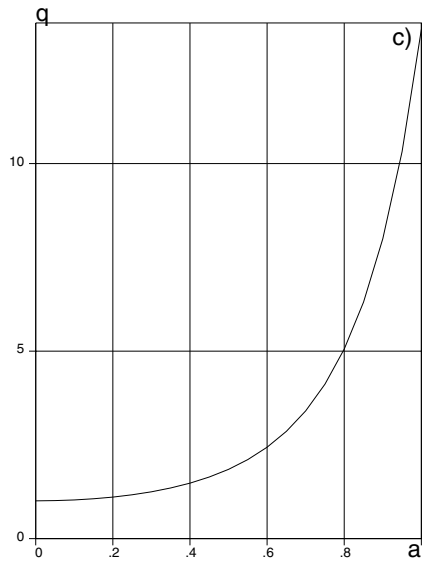

c)

Figure 1: Comparison of a small aspect ratio Soloviev equilibrium with an ESC, 5 harmonics calculation. a) Magnetic surfaces for equidistant $a=\sqrt{\bar{\Phi} / \bar{\Phi}_{b}}$, b) $p$ - pressure profile. Numerical and analytical solutions are indistinguishable. c) $q$ - profile as functions of $a$.

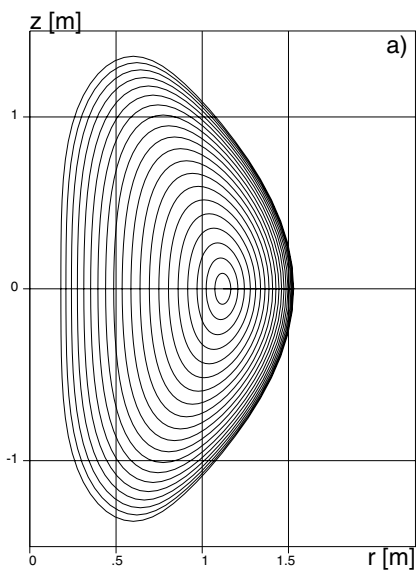

a)

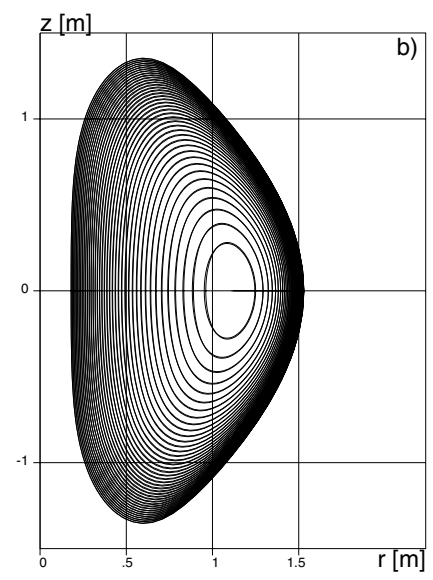

b)

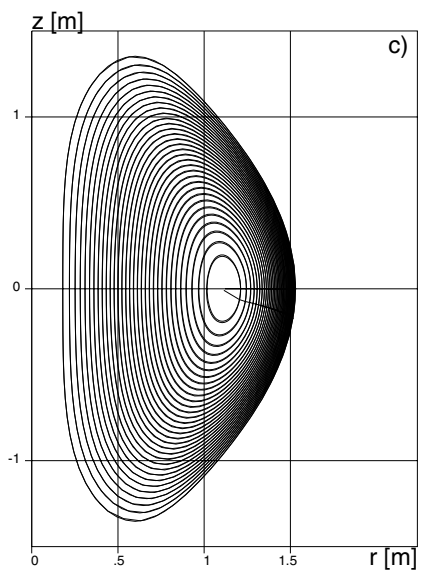

c)

Figure 2: Comparison of ESC calculations with the VMEC and JSOLVER codes. a) NSTX equilibrium configuration with $\beta=2 \mu_{0}\langle p\rangle / B_{\text {ext }}^{2}=8 \%$ ( $\langle p\rangle$ is the volume-averaged pressure, $B_{\text {ext }}=1 T$ is the vacuum toroidal magnetic field at the middle of the cross-section). Magnetic surfaces are shown for equidistant $a=\sqrt{\bar{\Phi} / \bar{\Phi}_{b}}$ b) ESC and VMEC magnetic surfaces are shown for equidistant $\bar{\Phi} / \bar{\Phi}_{b} ;$ c) ESC and JSOLVER magnetic surfaces are shown for equidistant $\bar{\Psi} / \bar{\Psi}_{b}$. 


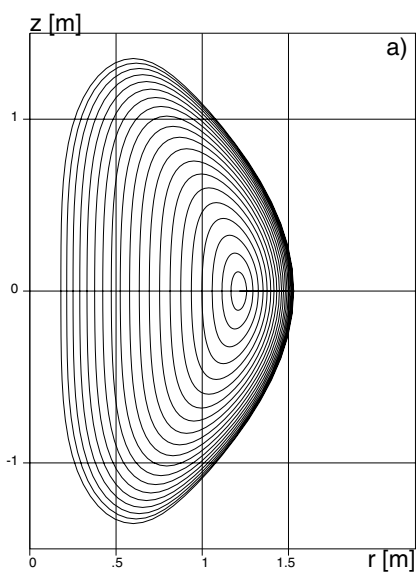

a)

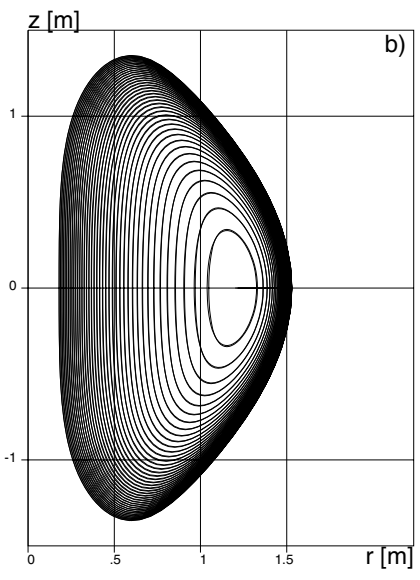

b)

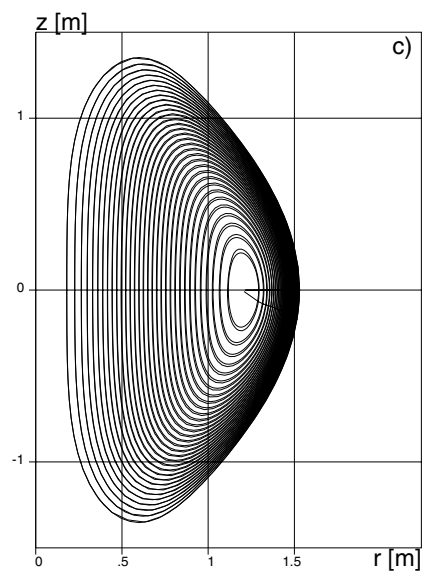

c)

Figure 3: Same comparison as in Fig. 2 but for $\beta=16 \%$.

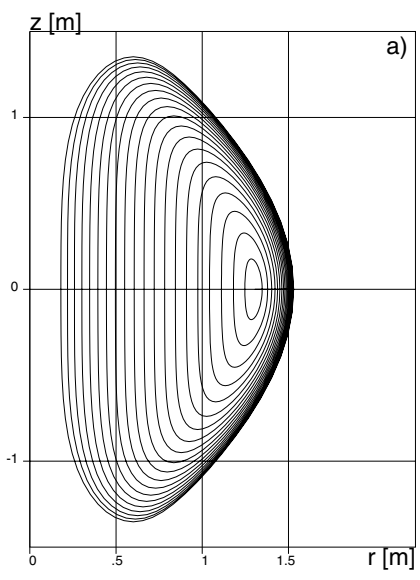

a)

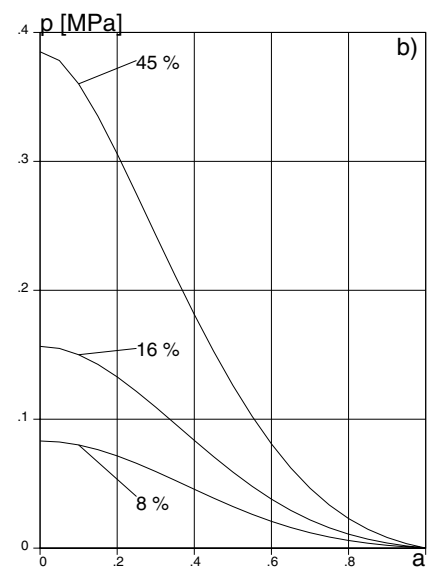

b)

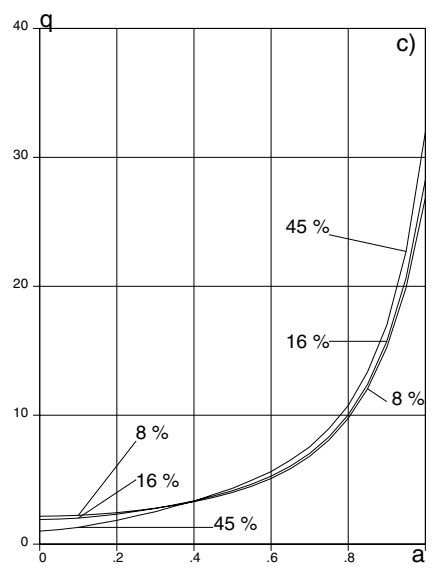

c)

Figure 4: a) Extreme NSTX magnetic configuration with $\beta=45 \%$; b) pressure and c) $q$-profiles as functions of $\sqrt{\bar{\Phi} / \bar{\Phi}_{b}}$ for Fig. 2,3,4a. 